\title{
Relativistic Quark-Model Spectroscopy of Light and Heavy Baryons
}

\author{
Joseph P. Day* \\ Theoretical Physics, Institute of Physics, University of Graz, A-8010 Graz, Austria \\ E-mail: joseph.dayeuni-graz.at
}

\section{Ki-Seok Choi}

Department of Physics, Soongsil University, Seoul 156-743, Republic of Korea

E-mail: kiseokchoi@gmail.com

\section{Willibald Plessas}

Theoretical Physics, Institute of Physics, University of Graz, A-8010 Graz, Austria

E-mail: willibald.plessas@uni-graz.at

\begin{abstract}
We report results from a study of light- and heavy-baryon spectra within a relativistic constituent quark model that relies on a universal dynamics for baryons of all flavor contents. The (linear) confinement is taken according to the string tension of quantum chromodynamics and the (flavordependent) hyperfine interaction is derived from an effective Lagrangian resulting from chiralsymmetry breaking. Here, we specifically show results for all baryons that are included in the phenomenological data base of the Particle Data Group with at least three-star status. In general, the spectra are reproduced in good agreement with experiment for both ground and excited states.
\end{abstract}

Sixth International Conference on Quarks and Nuclear Physics,

April 16-20, 2012

Ecole Polytechnique, Palaiseau, Paris

\footnotetext{
*Speaker.
} 


\section{Introduction and Motivation}

Quantum chromodynamics (QCD) is generally considered as the fundamental theory of strong interactions. While perturbation theory works well in the high-energy regime, QCD still lacks a comprehensive solution at low and intermediate energies. This is unfortunate because the wealth of phenomena and experimental data known very well lie in the non-perturbative regime. Over the last 40 years, since the inception of QCD, various approaches have been developed to study hadrons in the low- and intermediate-energy domain. Amongst them are lattice QCD, functional methods, effective field theories, chiral perturbation theory, to name only a few. In none of these approaches is the full dynamical content of QCD included. Basically, the difficulties are associated with a relativistically covariant treatment of confinement and the spontaneous breaking of chiral symmetry $(\mathrm{SB} \chi \mathrm{S})$, the latter being a well-established property of QCD at low and intermediate energies. As a result, most hadron reactions, like resonance excitations, strong and electroweak decays etc., are nowadays only amenable to models of QCD. Most famous is the constituent-quark model (CQM), which essentially relies on a limited number of effective degrees of freedom with the aim of encoding the essential features of low- and intermediate-energy QCD. Here, we discuss a relativistic constituent quark model (RCQM) that comprises all known baryons with flavors $u, d$, $s, c$, and $b$ within a single framework. It is constructed as an extension of the so-called Goldstoneboson-exchange (GBE) RCQM, which has so far been limited to $S U(3)_{F}$ baryons.

\section{Review of the $S U(3)_{F}$ GBE RCQM}

The GBE RCQM that has existed so far [1] covers baryons with flavors $u, d$, and $s$. The dynamical ingredients are constituent quarks subject to a linear confinement and interacting by a hyperfine interaction derived from Goldstone-boson exchange, the latter being realized by pseudoscalar meson exchange. It takes into account only the spin-spin part reading

$$
V_{i j}^{\mathrm{hf}}=\left[V_{\pi} \sum_{a=1}^{3} \lambda_{i}^{a} \lambda_{j}^{a}+V_{K} \sum_{a=4}^{7} \lambda_{i}^{a} \lambda_{j}^{a}+V_{\eta} \lambda_{i}^{8} \lambda_{j}^{8}+V_{\eta^{\prime}} \lambda_{i}^{0} \lambda_{j}^{0}\right] \vec{\sigma}_{i} \cdot \vec{\sigma}_{j}
$$

where the $\lambda_{i}^{a}$ are the $S U(3)_{F}$ Gell-Mann flavor matrices and $\vec{\sigma}_{i}$ the $S U(2)_{S}$ Pauli matrices of quark $i$. The various meson-exchange potentials are assumed in the following form as a function of the relative $Q-Q$ distance $\vec{r}_{i j}$

$$
V_{\gamma}\left(\vec{r}_{i j}\right)=\frac{g_{\gamma}^{2}}{4 \pi} \frac{1}{12 m_{i} m_{j}}\left[\mu_{\gamma}^{2} \frac{e^{-\mu_{\gamma} r_{i j}}}{r_{i j}}-\Lambda_{\gamma}^{2} \frac{e^{-\Lambda_{\gamma} r_{i j}}}{r_{i j}}\right], \quad \gamma=\pi, K, \eta, \eta^{\prime},
$$

where $g_{\gamma}$ is the meson-quark coupling constant, $\mu_{\gamma}$ the mass of the exchanged meson, and $\Lambda_{\gamma}$ an adjustable parameter in the regularizing cut-off. The GBE RCQM thus produces a spin- and flavordependent hyperfine interaction. Thereby it has proven quite successful not only in describing the spectroscopy of all baryons with flavors $u, d$, and $s$ in a unified framework but also with regard to a number of baryon reactions, such as electromagnetic and axial form factors, hadronic decays etc. For a recent summary of the GBE RCQM see, e.g., Ref. [2]. The GBE RCQM has also been extended to include beyond the spin-spin interaction of Eq. (2.1) all other force components stemming from GBE [3]. 
In Fig. 2 we demonstrate a typical and essential property of the GBE RCQM regarding the correct level orderings in the $N$ and $\Delta$ excitation spectra. The inversion of the $J^{P}=\frac{1}{2}^{+} N(1440)$ and $J^{P}=\frac{1}{2}^{-} N(1553)$ levels essentially comes about by the flavor-dependent hyperfine interaction. This is clearly visible in the right panel of Fig. 2, where the GBE interaction is gradually turned on starting from the case of confinement only to the size of the strength of the quark-meson coupling derived from the phenomenologically known $\pi-N$ coupling via the Goldberger-Treiman relation. For a detailed discussion of further properties of the GBE hyperfine interaction we refer to Ref. [4].
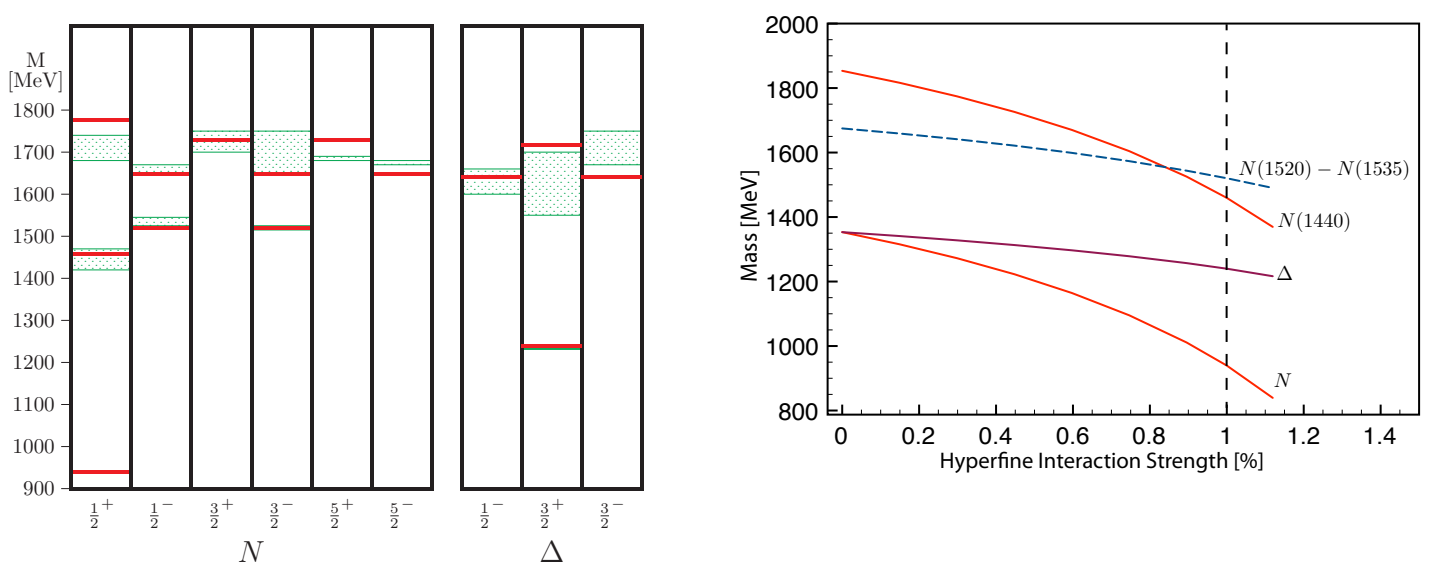

Figure 1: Left panel: Ground and resonance states (solid/red levels) as predicted by the GBE RCQM of Ref. [1] in comparison to experimental data and their uncertainties as compiled by the PDG [5] (green/shaded boxes). Right panel: Evolution of mass levels as a function of the strength of the hyperfine interaction.

\section{Universal GBE RCQM}

Led by the demand of a universal description of all existing baryons in single consistent framework and motivated by the successes of the previous GBE RCQM we have extended its construction to $S U(5)_{F}$. Close to a similar idea before by Glozman and Riska [6], we have thus advocated a hyperfine interaction of the following form

$$
V_{\mathrm{hf}}\left(\vec{r}_{i j}\right)=\left[V_{24}\left(\vec{r}_{i j}\right) \sum_{a=1}^{24} \lambda_{i}^{a} \lambda_{j}^{a}+V_{0}\left(\vec{r}_{i j}\right) \lambda_{i}^{0} \lambda_{j}^{0}\right] \vec{\sigma}_{i} \cdot \vec{\sigma}_{j}
$$

in order to include also charm and bottom baryons. Again, we take into account only the spin-spin component, which produces the most important hyperfine forces for the baryon spectra. The $\lambda_{i}^{a}$ are now the generalized Gell-Mann flavor matrices of $S U(5)_{F}$ for quark $i$. In addition to the exchange of the pseudoscalar 24-plet also the flavor singlet is included because of the $U(1)$ anomaly. The radial form of the GBE potentials resemble the one of Eq. (2.2) now reading

$$
V_{\beta}\left(\vec{r}_{i j}\right)=\frac{g_{\beta}^{2}}{4 \pi} \frac{1}{12 m_{i} m_{j}}\left[\mu_{\beta}^{2} \frac{e^{-\mu_{\beta} r_{i j}}}{r_{i j}}-\Lambda_{\beta}^{2} \frac{e^{-\Lambda_{\beta} r_{i j}}}{r_{i j}}\right], \quad \beta=0,24 .
$$

For all details of the construction and the selection of the parameters we refer to Ref. [7]. Here, we only stress that the UGBE RCQM has only three open parameters just as the previous $S U(3)$ GBE RCQM. They were determined by a best fit to the baryon spectra. 


\section{Spectra of the UGBE RCQM}

We have calculated the baryon spectra of the relativistically invariant mass operator $\hat{M}$ relying on the hyperfine interaction of Eq. (3.1) to a high accuracy both by the stochastic variational method [8] as well as the Faddeev integral-equation approach, modified for the treatment of (longrange) confinement problems $[9,10]$. The present UGBE RCQM produces the spectra in the light and strange sectors with similar quality as the previous GBE RCQM of Ref. [1] (see Figs. 2 and 3). Most importantly, the right level orderings specifically in the $N, \Delta$, and $\Lambda$ spectra as well as all other $S U(3)_{F}$ ground and excited states are reproduced in accordance with phenomenology. The reasons are exactly the same as for the previous GBE RCQM, which has already been extensively discussed in the literature $[1,4]$.
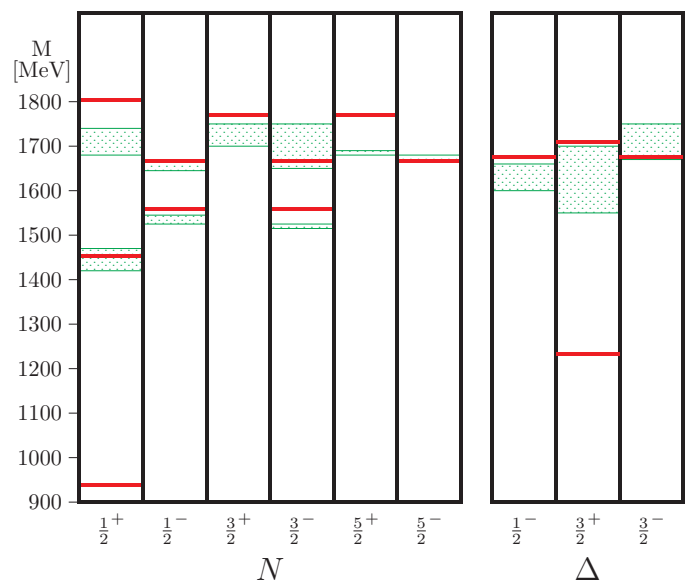

Figure 2: $N$ and $\Delta$ spectra as predicted by the UGBE RCQM discussed here. The solid/red lines represent the theoretical levels, the green/shaded boxes the experimental data with uncertainties as compiled by the PDG [5].
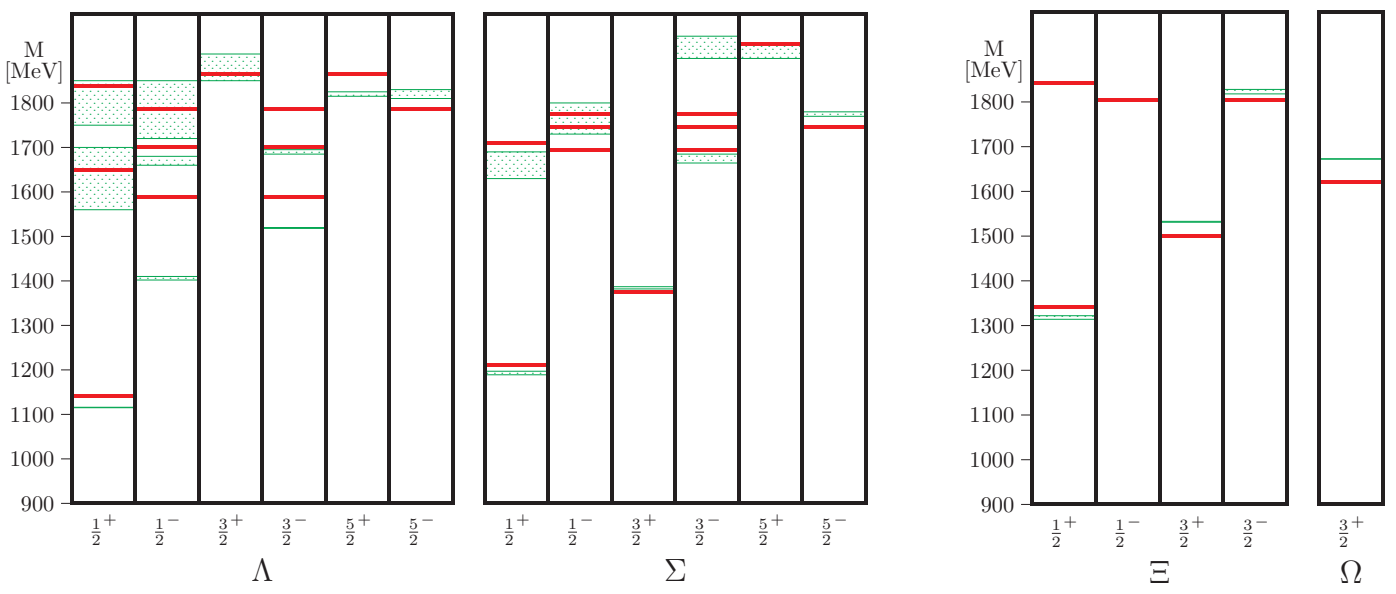

Figure 3: Same as Fig. 2 but for the hyperons. 
By means of the UGBE RCQM we are in the position to study also the light-heavy and heavy-heavy $Q-Q$ hyperfine interactions, which are so far largely unexplored. At least there is still a considerable theoretical uncertainty, which kind of hyperfine interaction, gluon exchange or Goldstone-boson exchange, is prevailing. Here we show that GBE dynamics is also appropriate for the hyperfine interaction between light-heavy as well as heavy-heavy constituent quarks. Specifically, the spectra of all charm and bottom baryons can be produced in accordance with existing experimental data for states rated with at least three- or four-stars by the PDG (see Figs. 4 and 5) ${ }^{1}$.
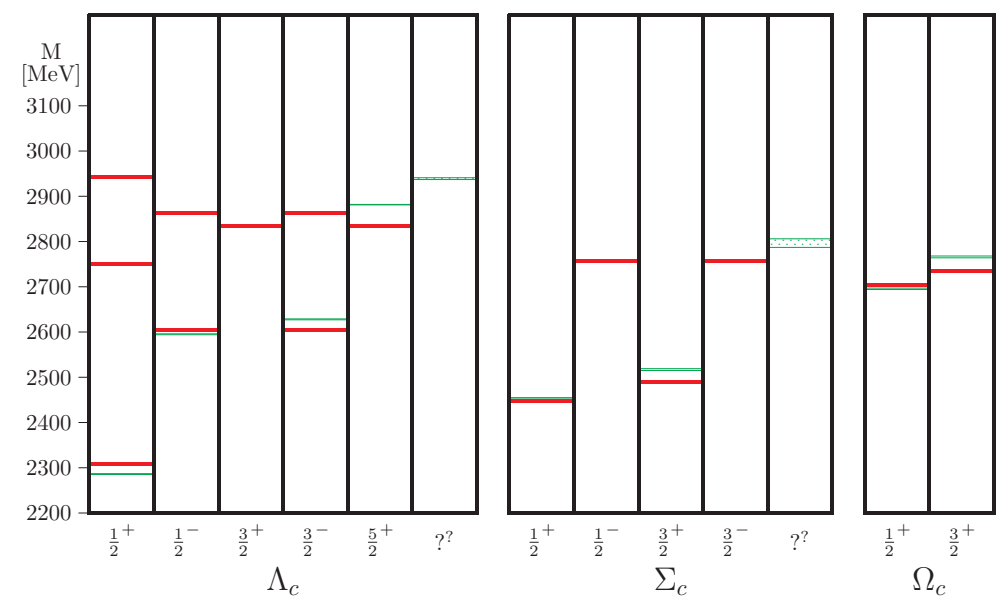

Figure 4: Spectra of single-charm baryons as predicted by the UGBE RCQM discussed here. Same notation as in Fig. 2.
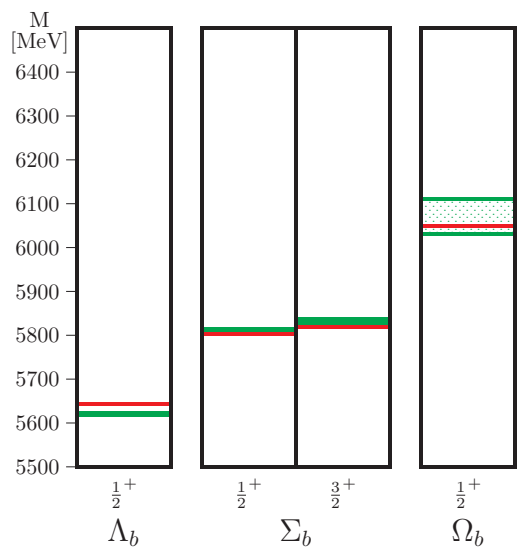

Figure 5: Spectra of single-bottom baryons as predicted by the UGBE RCQM discussed here. Same notation as in Fig. 2.

Of course, the presently available experimental data base on charm and bottom baryons is not yet very rich and thus not particularly selective for tests of different $Q-Q$ hyperfine interactions. The

\footnotetext{
${ }^{1}$ Only the $\Xi_{c}$ and $\Xi_{b}$ states are so far missing in our analysis, as we are presently not in the position to calculate baryons with three different constituent-quark masses.
} 
situation will certainly improve with the advent of further measurements in ongoing and planned experiments. Beyond experimental data, we may also compare our results to lattice-QCD calculations and predictions by other theoretical models. Without dwelling on further details here, due to space limitations, we note that the heavy-baryon spectra produced by the UGBE RCQM are specifically in good agreement with results from refined lattice-QCD calculations that have now become available. This is especially true for the charm baryons vis-à-vis the recent work by Liu et al. [11]. Detailed comparisons with results from several lattice-QCD and alternative methods will be given in a forthcoming more comprehensive article on the UGBE RCQM [12]. There we shall show also a number of additional theoretical spectra up to $\Omega_{b b b}$ (for which, however, no phenomenological data exist so far).

\section{Conclusion}

We have shown that it is possible to describe the entire spectroscopy of known baryons within a single framework of a RCQM in reasonable agreement with phenomenology. In addition, as shown in the conference presentation, the new UGBE RCQM is able to make predictions for double- and triple-heavy baryons that are not yet detected. In future it will be interesting to see how the UGBE RCQM will perform in several applications, such as electroweak baryon form factors and other similar observables testing the corresponding baryon states.

\section{Acknowledgement}

This work was supported by the Austrian Science Fund, FWF, through the Doctoral Program on Hadrons in Vacuum, Nuclei, and Stars (FWF DK W1203-N16).

\section{References}

[1] L. Y. .Glozman, W. Plessas, K. Varga, and R. F. Wagenbrunn, Phys. Rev. D 58, 094030 (1998)

[2] W. Plessas, PoS LC 2010, 017 (2010); [arXiv:1011.0156]

[3] K. Glantschnig, R. Kainhofer, W. Plessas, B. Sengl, and R. F. Wagenbrunn, Eur. Phys. J. A 23, 507 (2005)

[4] L.Ya. Glozman, Z. Papp, W. Plessas, K. Varga, and R.F. Wagenbrunn, Phys. Rev. C 57, 3406 (1998)

[5] K. Nakamura et al. [Particle Data Group Collaboration], J. Phys. G 37, 075021 (2010)

[6] L.Ya. Glozman and D. O. Riska, Nucl. Phys. A 603, 326 (1996) [Erratum ibid. A 620, 510 (1997)]

[7] J. P. Day, W. Plessas, and K. -S. Choi, arXiv:1205.6918 [hep-ph].

[8] Y. Suzuki and K. Varga, Stochastic Variational Approach to Quantum-Mechanical Few-Body Problems, Lecture Notes in Physics 54, 1 (1998)

[9] Z. Papp, A. Krassnigg, and W. Plessas, Phys. Rev. C 62, 044004 (2000)

[10] J. McEwen, J. Day, A. Gonzalez, Z. Papp, and W. Plessas, Few-Body Syst. 47, 225 (2010)

[11] L. Liu, H. -W. Lin, K. Orginos, and A. Walker-Loud, Phys. Rev. D 81, 094505 (2010) [arXiv:0909.3294 [hep-lat]].

[12] Joseph P. Day, Ki-Seok Choi, and Willibald Plessas, to be published 